\title{
Aclidiniumbromid hält COPD-Symptome in Schach
}

- Mit Aclidiniumbromid - angeboten als Eklira ${ }^{\circledR}$ Genuair $^{\circledR}$ von Almirall und Bretaris ${ }^{\circledR}$ Genuair $^{\oplus}$ von Berlin-Chemie - steht in Deutschland seit Juli 2012 ein langwirksamer selektiver Muskarin-Rezeptor-Antagonist (LAMA) zur Verfügung. Nach der Inhalation wird er rasch in inaktive Metabolite hydrolysiert, sodass geringe Plasmakonzentrationen der Substanz resultieren. Damit sind kaum systemische anticholinerge Wirkungen zu erwarten.

Erwachsene mit COPD nehmen zur Linderung ihrer Symptome zweimal täglich $375 \mu \mathrm{g}$ Aclidiniumbromid (entspricht $322 \mu \mathrm{g}$ Aclidinium) mit Hilfe des Multidosis-Pulverinhalators (Genuair ${ }^{\circledast}$ ) ein. So wird eine Symptomkontrolle über 24 Stunden ge- währleistet, die mit der Wirkung des derzeitigen Therapiestandards Tiotropium vergleichbar ist. Dabei ist die Pharmakokinetik der Substanz unabhängig von der Nierenfunktion. Bei Patienten mit eingeschränkter Nierenfunktion ist somit keine Dosisanpassung erforderlich.

In klinischen Studien wurde die Wirkung von Aclidiniumbromid auf die Lungenfunktion im Vergleich zu Placebo sowie im Vergleich zu Tiotropium geprüft. Aclidinium gilt als sicher und gut verträglich. Häufigste Nebenwirkungen in den Studien waren Kopfschmerzen $(6,6 \%)$ und Nasopharyngitis $(5,5 \%)$. Anticholinerge Effekte waren ebenso häufig wie unter Placebo. Gesunden Probanden hatten inhalierte Einzeldosen von bis zu $6000 \mu \mathrm{g}$ Aclidiniumbromid erhalten, ohne dass anticholinerge Effekte auftreten waren. Auch zweimal täglich $800 \mu \mathrm{g}$ Aclidiniumbromid über sieben Tage führte zu keinen klinisch relevanten Nebenwirkungen.

\section{Weniger Symptome frühmorgens}

Im Direktvergleich mit Tiotropium (18 $\mu \mathrm{g}$ einmal täglich) und Placebo waren die bronchodilatatorischen Effekte ähnlich gut und effektiver als bei Placebogabe. Die nächtliche Lungenfunktion konnte numerisch mit Aclidinium am stärksten verbessert werden: Die COPD-Symptome nahmen nachts auf einer Fünf-Punkte-Skala signifikant um 0,16 Punkte ab (Placebo: -0,02), unter Tiotropium um 0,09 Punkte. Dr. Thomas Meißner .

\section{$-$ Brentuximab Vedotin gegen Lymphome \\ Patienten mit rezidiviertem/refraktärem ( $r / r)$ Hodgkin-Lym- phom (HL) oder systemischem anaplastischen großzelligen Lymphom ( $\mathrm{SALCL}$ ) haben eine ungünstige Prognose. Mit dem Antikörper-Wirkstoff-Konjugat Brentuximab Vedotin (Adcetris ${ }^{\circledR}$ ) von Takeda steht seit zwanzig Jahren erstmals wieder eine Sub- stanz zur Verfügung, die das Potenzial hat, die Prognose der Pa- tienten zu verbessern. \\ Das Besondere an Brentuximab Vedotin ist der innovative Wirk- mechanismus, der eine gezielt gegen die Tumorzellen gerich- tete antineoplastische Wirksamkeit bei gleichzeitig geringer systemischer Belastung ermöglicht.}

Birgit-Kristin Pohlmann -

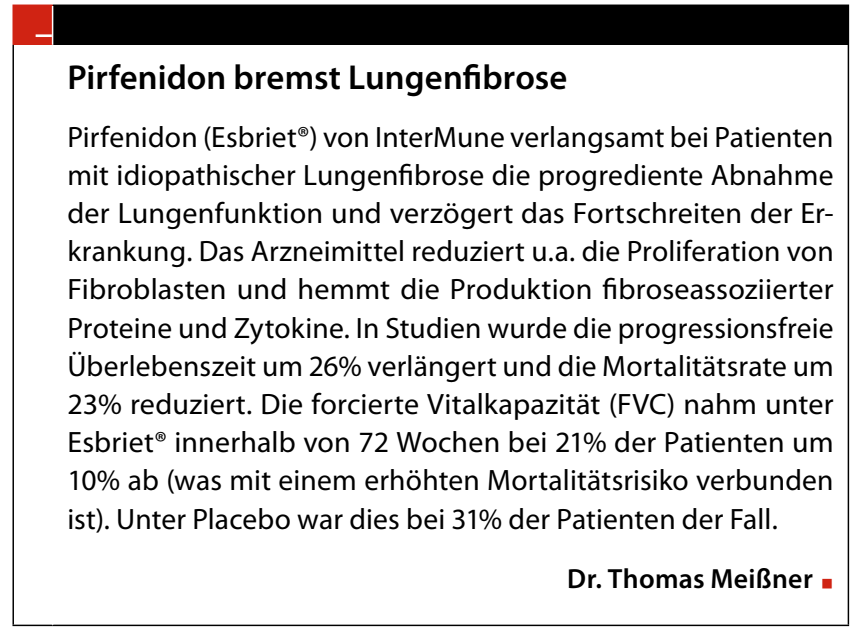

\section{Ivacaftor: Kausale Waffe bei zystischer Fibrose}

Der selektive CFTR-Potenziator Ivacaftor $\left(\right.$ Kalydeco $\left.^{\mathrm{TM}}\right)$ von Vertex Pharmaceuticals ist ein kausal wirkendes orales Medikament für Kinder ab sechs Jahren und Erwachsene. Ivacaftor steigert die Funktion des Chloridionentransportkanals exokriner Drüsen. Die gesteigerte Chloridkonzentration in der Flüssigkeitsschicht der Atemwege sorgt dafür, dass mehr Wasser in den Sekreten gehalten werden kann, weshalb die Sekrete flüssiger werden. Das ist vor allem für die mukoziliäre Clearance bedeutsam. Innerhalb von zwei Wochen bessert sich nachhaltig die Lungenfunktion. Die behandelten Patienten nehmen an Körpergewicht zu und die Lebensqualität steigt.

\section{Dr. Thomas Meißner =}

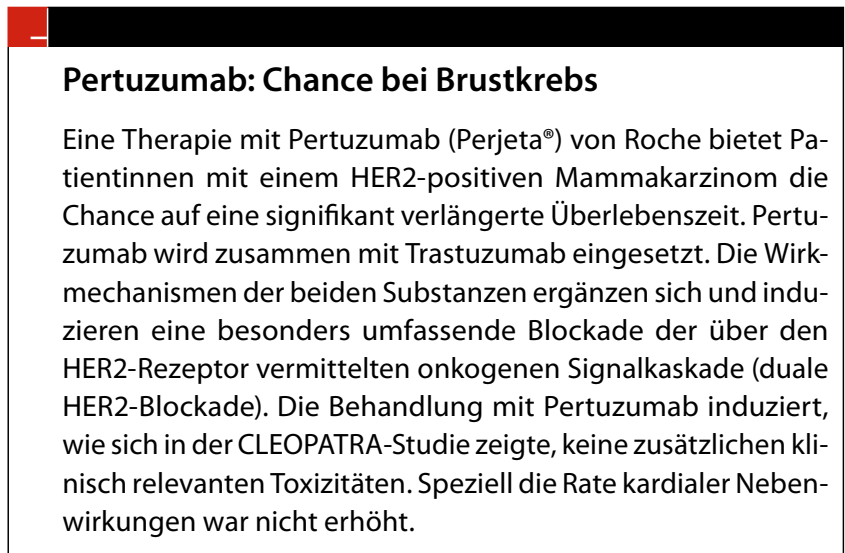

Birgt-Kristin Pohlmann . 